\title{
Critical Analysis of Data Forwarding Routing Protocols Based on Single Path for UWSN
}

\author{
Mukhtiar Ahmed $^{1,2}$, Mazleena Salleh ${ }^{1}$, M. Ibrahim Channa ${ }^{2}$ \\ ${ }^{1}$ Departement of Computer Science, Faculty of Computing, UTM, Malaysia \\ ${ }^{2}$ Departement of Information Technology, Faculty of Science, QUEST, Nawabshah, Sindh, Pakistan
}

\begin{abstract}
Article Info
Article history:

ABSTRACT
In Underwater Wireless Sensor Network (UWSN); the sensor node is
responsible to extract the valuable application based information from
underwater environment. The application based information covers the
applications like: tactical surveillance, assisted navigation, disaster
prevention, offshore exploration, pollution monitoring and oceanographic
data collection. The design of routing protocol in underwater environment is
one of the challenging issues for researchers. This research article focuses the
designing issues of the data forwarding routing protocols based on single
path. In this article the designing of $2 \mathrm{D}$ and $3 \mathrm{D}$ architecture of routing
protocols are discussed with their different issues. This article also focuses
the analytical approach of proposed routing protocols with different
parameters, the simulation scenarios of the single path routing protocols with
critical analysis; and the open research issues; will help the researchers to
further research in the field of routing protocols for UWSN.

Received Feb 17, 2016

Revised May 26, 2016

Accepted Jun 10, 2016

\section{Keyword:}

Courier node

Deployment

Location-aware

Propagation delay

Route discovery
\end{abstract}

Copyright (c) 2016 Institute of Advanced Engineering and Science. All rights reserved.

\section{Corresponding Author:}

Mukhtiar Ahmed,

Department of Computer Science, Faculty of Computing,

University Technology Malaysia (UTM),

Skudai Johar Malaysia,

Email: mukhtiar.a@gmail.com

\section{INTRODUCTION}

Majority of the researchers are well interested in the field of the Underwater Wireless Sensor Network (UWSN) due to its well prominent applications like disaster prevention tactical underwater surveillance, assisted navigation, pollution tracking, marine climate observation, extraction of underwater minerals, information about underwater mountains and underwater wild life [1]. To extract the information from the underwater environment the wireless sensor nodes are the source. Majority of the researchers have used the terrestrial network approach for designing of routing strategies in underwater environment like: some researchers have used the supporting QoS is an important task in routing protocols [2]. This includes real-time communication, reliable transmission, and resource reservation. Packets should be transmitted as soon as possible over the most reliable link while considering bandwidth constraints [2]. Others researchers have used the MANET routing protocol approach for the movement of the node, the node in MANET moves arbitrary which may experience rapid and unpredictable changes in the network topology [3]. Others researchers have used the approach of the LEACH, DSDV and OLSR routing protocol for data forwarding [4]-[6]. The deployment of wireless sensor nodes in underwater environment is one of the challenging issues because the pressure of the underwater is the major hurdle for deployment and a fixed or static architecture is not appropriate in underwater environment due to the continuous movement of the water [7]-[9]. In underwater environment the dynamic architecture is the best solution because node moves with respect to the water pressure [10],[11]. Majority of the researches have designed the location based and localization free network architecture but still the research is needed for some serious issues like: (i) in underwater the 
acoustic channel has a limited bandwidth, (ii) acoustic channel is impaired with multipath and fading, (iii) propagation delay due to the acoustic channel, (iv) there is high bit error rate and temporary loss connectivity due to void regions, (v) batteries of the sensor nodes cannot be recharged easily and (vi) sensor nodes may dead due to fouling and corrosion. The researchers are also involved to solve the localization and deployment problems [12]. This research article focuses the critical analysis of the routing protocols for underwater wireless sensor network which are based on single path data forwarding. This research article focuses the network architecture, deployment of sensor nodes, route discovery mechanism, data forwarding, and route maintenance mechanism. The major part of this article focuses the issues with the proposed routing protocols and the comparative and critical analysis on analytical and simulation results of the proposed routing protocols. This article will help the researchers to further research on proposed routing protocols.

\section{BACKGROUND AND LITERATURE REVIEW}

Similar to terrestrial networks, underwater wireless sensor network (UWSN) also refers the variable number of sensor nodes with different names like autonomous underwater vehicles, moored sensors, acoustic sensors, relay nodes; super nodes cabled sea floor sensors and courier nodes. Majority of the researchers have worked on the routing protocols for underwater wireless sensor networks. Some researchers have designed cluster based routing protocols, some have designed geographical based routing protocols, some have worked on energy efficient routing, some have designed multi-path data forwarding, some have designed vector based and some have designed the single path data forwarding routing protocols for underwater wireless sensor networks. This article focuses the routing protocols based on single path; the famous routing protocols are:

i. Information-Carrying Routing Protocol (ICRP)

ii. Resilient Routing Algorithm

iii. Underwater Wireless Hybrid Sensor Networks (UW-HSN)

iv. Location-Aware Source Routing (LASR)

v. Delay-tolerant Data Dolphin (DDD)

\subsection{Information-Carrying Routing Protocol (ICRP)}

In [13] the Information-Carrying Routing Protocol (ICRP) is proposed for underwater wireless sensor network. ICRP is data forwarding routing protocol which is based on single path; ICRP is an energy efficient, scalable and real time localization free routing protocol. In ICRP the source node is responsible for route discovery through route discovery message. On arrival of route discovery message the intermediate or neighbor nodes will establish the reverse route for acknowledgement. When route established the source will forward the packets and will wait for acknowledgment through reverse route. The established routes refer the TIMEOUT function, if the threshold time exceeds the TIMEOUT than route become invalid. When the data packets received through the established route to the destination the delivery refers the successful packets delivery.

Issues with ICRP: (i) ICRP node mobility model is invalid due to water pressure. (ii) ICRP Route information and TIMEOUT function mechanisms may drop the packets due to void regions. (iii) ICRP refers the route validity for long or short time which will affect the data delivery ratio because in underwater environment the node movement is only for 2 to $3 \mathrm{~m} / \mathrm{sec}$.

\subsection{Resilient Routing Algorithm}

The Resilient algorithm for long-term applications is the single path data forwarding routing algorithm [14]. The authors have considered the three major issues like: limited bandwidth, temporary loss of connectivity and node failure for the designing of resilient algorithm. The routing algorithm completes its task into two phases. In first phase authors have discovered the backup multi-hop and primary optimal node disjoint methods to minimize the energy consumption of the sensor nodes. In the second phase the authors have introduced the online distributed scheme which specially observes the network; if there is any problem in network or may be the failure of any single or multiple devices than the scheme allows switching to the backup path because in underwater environment the monitoring mission is highly expensive.

The Resilient architecture refers the winch-based sensor devices; which are fixed at the sea bottom. Each sensor device is equipped with a floating buoy that can be adjusted by a pump. The buoy is a source to push the sensor device towards the surface level of water. The electronic controlled engine is fixed into the sensor device. Electronic controlled engine is used to control the depth of the device through the adjusting of the length of wire. Issues with Resilient: (i) Resilient scheme is only for the long-term applications. (ii) Resilient overall network cost is high. 


\subsection{Underwater Wireless Hybrid Sensor Networks (UW-HSN)}

The authors have considered the issues in shallow water like: large propagation delays, high signal attenuation, low bandwidth and energy consumption for the designing of Underwater Wireless Hybrid Sensor Networks (UW-HSN) [15]. For large and continuous communications the authors have used the radio and acoustic signaling system. The sensor nodes are equipped with the acoustic and radio modem; in underwater the sensor node uses the acoustic signaling whereas on surface level the sensor node uses the radio signaling to communicate with base station. The sensor node is also equipped with mechanical module which forces the sensor node swim on surface and dive back to the different levels of water. The authors have used the TurtleNet hybrid concept for negative and positive vertical movements of the node through piston to reach on surface and dive back to the bottom depth levels of water. The Trutle Distance Vector (TDV) algorithm determines the communication channel in order to minimize average event delay. Term event delay means successful reception time duration between source nodes and base station.

Issues with UW-HSN: (i) UW-HSN energy consumption model and parameters are not properly defined. (ii) The hardware used by UW-HSN increases the overall cost of the network.

\subsection{Location-Aware Source Routing (LASR)}

Location-Aware Source Routing (LASR) is the modified version of the Dynamic Source Routing (DSR) proposed by [16]. The authors have considered the high latency problem in underwater environment and designed the LASR protocol to resolve the problem. LASR is based on two techniques to resolve the high latency problem; the first is link quality and the second is location awareness. DSR protocol is for shortest path but LASR rely on better routes throughout the network by using the Expected Transmission Count (ETX); which is better for the link quality. The ETX can be calculated as given in Equation (1).

$$
\mathrm{ETX} \triangleq \frac{1}{(1-\pi I R)^{2}}
$$

In Equation (1) FER denotes the Frame Error Rate. The link quality protocol header is consists on octal 12-bit. The time stamp factor is used for new data link. LASR also guarantees for state less link type data which correctly be discarded through some mechanism.

The route can be replaced when the implicit information appears to build the link cache. LASR has used the Dijkstra's algorithm for updating the network graph. Route handling mechanism will use the protocol options to develop the route link, protocol options are acknowledgement, route selection, and route reply. LASR has used the three features when transmits the number of packets on route, these features are acknowledgment delay guarantee, hello message and option packing. The authors claimed that LASR increases the packets delivery ratio and reduces the end to end delay. The LASR Tracking System is recursive and state-estimation filter that uses the range estimates to predict network topology. Its performance is modeled.

Issues with LASR: (i) LASR have inherited the routing mechanism from the DSR and if the hop counts between source and destination increases the size of the packets' header also increases and in resultant the overheads increases with narrow band for communication in underwater. (ii) LASR uses Expected Transmission Count (ETX) as a link quality metric, for which it assumes that links are symmetrical and are with the same link quality in both directions, which is not easily possible for underwater acoustic communication [17].

\subsection{Delay-tolerant Data Dolphin (DDD)}

Delay-tolerant Data Dolphin (DDD) is an energy efficient routing scheme proposed by [18]. DDD is for the delay tolerant applications. The DDD routing scheme is based on collector nodes called dolphin and stationary nodes; the dolphin nodes harvest the information sensed by the stationary nodes. The routing scheme eliminates the energy expensive multi-hop communication. The stationary nodes are responsible to transmit its collected data to the nearest in range dolphins. The stationary nodes are deployed on sea bed area of interest. The acoustic modem through its communication component communicate to the dolphin nodes and low power transceivers component of acoustic modem analyzes the presence of in range dolphin nodes through the beacon signal generated by dolphin nodes. The dolphin nodes transfers the collected packets to the base station deployed on water surface.

Issues with DDD: (i) In DDD the random movement of dolphin nodes will not able to collect all the data packets from the sensor nodes and in resultant the data delivery ratio will be degraded. (ii) In DDD; if we increase the number of dolphin nodes the overall cost of the network will also be increased. 


\section{ANALYSIS AND DISCUSSION}

In this section we have evaluated the single path routing protocols through analytical method and simulation numerical method.

\subsection{Analytical Method}

Through this method we have analyzed the proposed routing protocols through performance metric and network parameters. The performance metrics parameters are: performance, cost efficiency, data delivery, energy efficiency, bandwidth, and reliability. The comparison through performance metric is shown in Table 1. Table 2 focuses the comparison of proposed routing protocols through network architecture parameters.

Table 1. Comparison of Routing Protocols through Performance Metrics

\begin{tabular}{lccccccc}
\hline Protocol & Performance & $\begin{array}{c}\text { Cost } \\
\text { Efficiency }\end{array}$ & $\begin{array}{c}\text { Data } \\
\text { Delivery }\end{array}$ & $\begin{array}{c}\text { Delay } \\
\text { Efficiency }\end{array}$ & $\begin{array}{c}\text { Energy } \\
\text { Efficiency }\end{array}$ & $\begin{array}{c}\text { Bandwidth } \\
\text { Efficiency }\end{array}$ & Reliability \\
\hline ICRP & Low & High & Low & Low & Medium & Medium & Low \\
Resilient RA & Medium & Low & High & Low & Medium & Medium & High \\
UW-HSN & Low & Low & Medium & High & Low & Medium & Medium \\
LASR & Medium & High & Medium & Low & Medium & Medium & Medium \\
DDD & Low & Low & Low & Low & High & Medium & Medium \\
\hline
\end{tabular}

Table 2. Comparison of routing protocols through Network Architecture Parameters.

\begin{tabular}{lcccccc}
\hline Protocol & $\begin{array}{c}\text { Single/ } \\
\text { Multiple } \\
\text { Copies }\end{array}$ & $\begin{array}{c}\text { Hop-by- } \\
\text { Hop/ End- } \\
\text { to-End }\end{array}$ & $\begin{array}{c}\text { Routing } \\
\text { Approach }\end{array}$ & $\begin{array}{c}\text { Single/ } \\
\text { Multi-sink }\end{array}$ & $\begin{array}{c}\text { Hello } \\
\text { Msg }\end{array}$ & $\begin{array}{c}\text { Localization } \\
\text { Needed }\end{array}$ \\
\hline ICRP & Multiple & End-to-End & Path-Based & Single & No & No \\
Resilient RA & Single & End-to-End & Path-Based & Single & No & Yes \\
UW-HSN & Single & Hop-by-Hop & Vector-Based & Single & Yes & No \\
LASR & Single & End-to-End & Path-Based & Single & Yes & No \\
DDD & Single & Single Hop & Path-Based & N/A & Yes & No \\
\hline
\end{tabular}

\subsection{Simulation Numerical Method}

This method focuses the simulation scenarios of the proposed routing protocols. Table 3 refers the generalized simulation parameters used by NS2.30 with AquaSim features.

Table 3. Simulation parameters used by NS2.30

\begin{tabular}{lclc}
\hline \multicolumn{1}{c}{ Parameter } & Rating & \multicolumn{1}{c}{ Parameter } & Rating \\
\hline Network Size & $500 \times 500 \times 50 \mathrm{~m}^{3}$ & Acoustic and Radio Data Rate & $30 \mathrm{~Kb} / \mathrm{s}$ and $1 \mathrm{Mb} / \mathrm{s}$ \\
No. of Nodes & 100 & Nakagami- $m$ (Best-condition) & 2.0 \\
Bandwidth & $50 \mathrm{Kbps}$ & PSD (Best-condition) & $55.0 \mathrm{~dB} / \sqrt{\mathrm{Hz}}$ \\
Communication Rate & $600 \mathrm{bps}$ & Nakagami- $m$ (Worst-condition) & 1.5 \\
Transmission power & $1 \mathrm{~W}$ & PSD (Worst-condition) & $65.0 \mathrm{~dB} / \sqrt{\mathrm{Hz}}$ \\
Packets Size & 256 & Channel Frequency & $50 \mathrm{KHz}$ \\
Packets interval arrival time & $600 \mathrm{~s}$ & Dolphin constant speed & $5 \mathrm{~m} / \mathrm{sec}$ \\
Contention Window & $8-64$ & Buffer Size & $1-500$ \\
\hline
\end{tabular}

Figure 1 refers the simulation scenario of ICRP in which the time in hours versus delay in seconds is focused. In the scenario the distance between nodes is settled $1600 \mathrm{~m}$, communication rate is $600 \mathrm{bps}$, modem frequency is $9-14 \mathrm{KHz}$, Data packet size is 112 Bytes, control packet size is 16 Bytes, average transmission energy is $40 \mathrm{~W}$ and relative moving speed between nodes is from 0 to $1.5 \mathrm{~m} / \mathrm{sec}$. The graph shows end-to-end delay.

Critical Analysis on ICRP: When we increase the number of nodes from 100 to 200 or 300 the endto-end delay will be increases. ICRP response in underwater environment is not reasonable. Figure 2 focuses the simulation scenario of Resilient routing protocol which shows Time in seconds versus Packets Delay in seconds. In graph the time increases the delay in packets also is increases.

Critical Analysis on Resilient: The result of Resilient routing algorithm is tested only on 50 number of nodes deployed in underwater environment; if we increase the number of nodes from 100 to 300 than the simulation results shows the larger average delay. The routing algorithm is only for long term applications. Figure 3 focuses the simulation scenario of UW-HSN; the Time in seconds versus Good put in Kilobits. The 
simulation result is for the TurtleNet and in article the result is compared with all-acoustic schemes and authors shows that the TurtleNet throughput is better than other schemes; even all other schemes comparison is questionable.

Critical Analysis of UW-HSN: The authors of the UW-HSN focuses the 2D deployment and almost the methodology focuses the terrestrial networks; even the response of the results is not good enough in comparison other UW routing schemes. The simulation scenario is also focuses the fewer throughputs according to the number of nodes deployed in the deployment area. Methodology and simulation results need to be improvement.

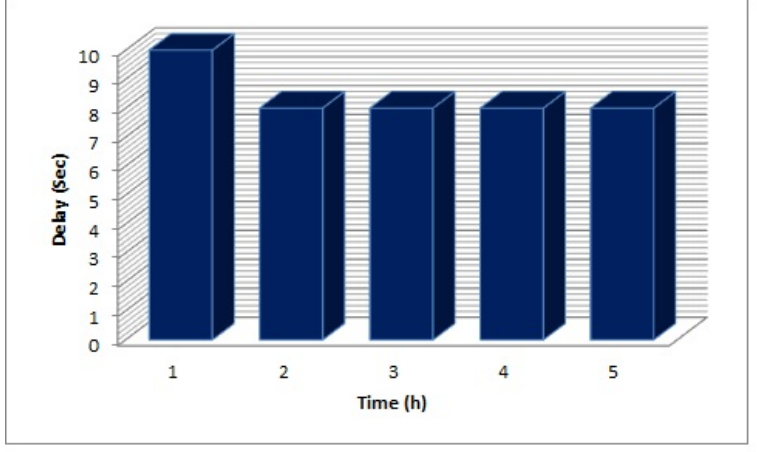

Figure 1. ICRP Time versus delay

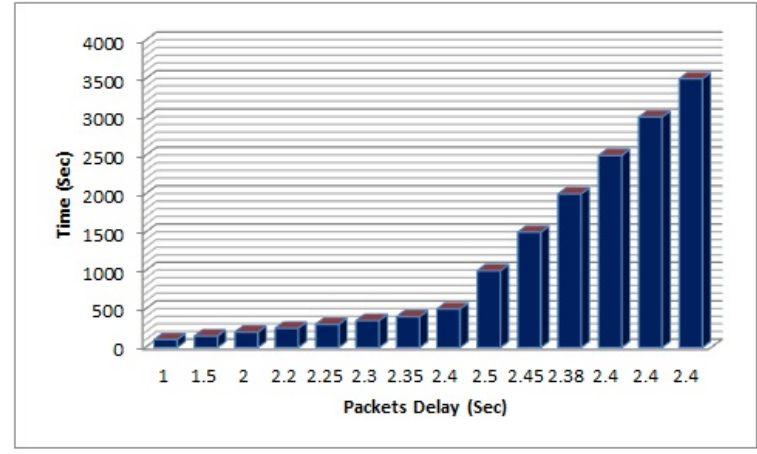

Figure 2. Resilient Packets Delay versus Time

Figure 4 is for LASR routing protocols which focuses the simulation result based on time in sec versus average network capacity in bits/sec. The network capacity has been measured on 20 simulation runs and average calculation is $300 \mathrm{sec}$.

Critical Analysis of LASR: The authors have considered the most of the parameters related to the terrestrial network; in the research article the authors have compared the results with the DSR protocol; this is terrestrial network protocol. The simulation scenario only focuses the fixed network capacity with respect to time; but in underwater environment the dynamic network capacity parameters should be considered.

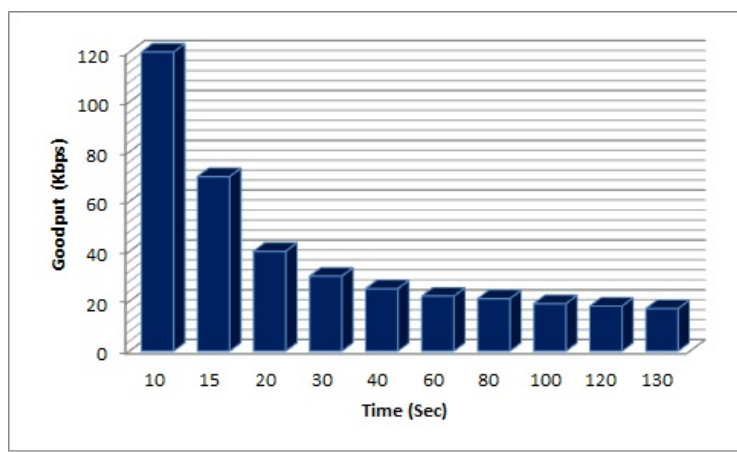

Figure 3. UW-HSN Time versus Good put

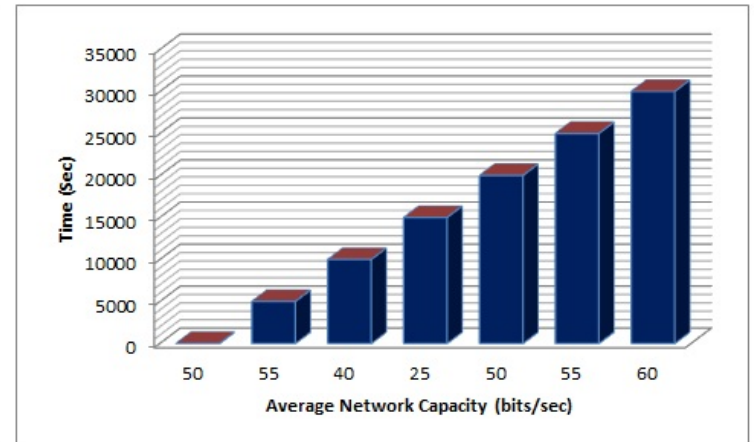

Figure 4. LASR Avg. N/W capacity versus Time

Figure 5(a) and Figure 5(b) focuses the simulation scenario of DDD routing protocol and simulation scenario is based on number of dolphins versus normalized delay according to buffer size. The scenario focuses as the number of dolphin increases the normalized delay decreases.

Critical Analysis on DDD: The simulation is based on the increased number of dolphins and ultimately the cost of overall network will also be increases. Dolphin constant speed is $5 \mathrm{~m} / \mathrm{sec}$ and the sensor nodes movement is 2 to $3 \mathrm{~m} / \mathrm{sec}$ it means that if dolphin remains away from the nodes than ultimately the delay will be increases and the overall throughput also be affected. The authors have not discussed the removal of void regions in the article; there are many more void regions are present in the underwater environment; so ultimately if we cannot consider removal of void regions the overall packets delivery ratio will also be affected. 
Figure 6 shows the simulation scenario of proposed routing protocols; the scenario focuses the number of nodes versus data delivery ratio. Figure7 shows the simulation scenario of the proposed routing protocols; the scenario focuses the number of nodes versus energy consumptions in joules. We have selected random number of nodes like: $15,20,25,30,35,40,45$ and 50 and tested the average results for data delivery ratio and in energy consumption. In Figure 6 for example if we consider the numbers of nodes are 20 than the data delivery ratio for Resilient is $98 \%$; whereas the data delivery ratio for DDD is $87 \%$. For energy consumption if we consider the number of nodes 20 the average energy consumption for Resilient is 850 joules and for DDD is 700 joules. From the simulation scenarios it is clear that Resilient Routing Algorithm is better in performance than other routing protocols based on single path. The only drawback in Resilient is that it is only for long-term applications and its overall network cost is high.
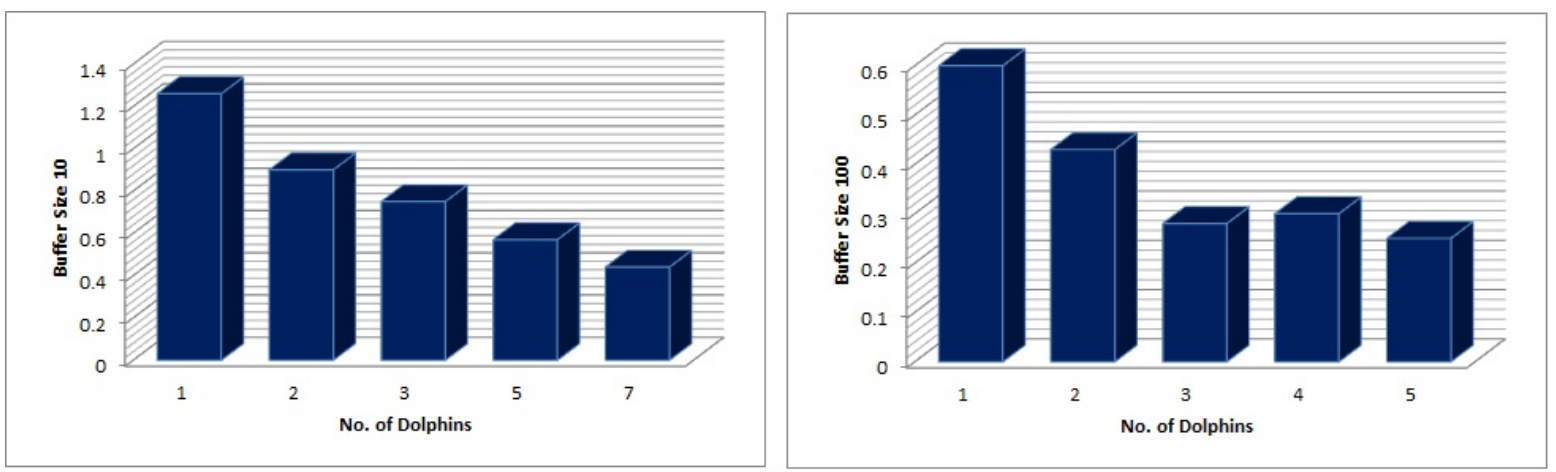

Figure 5a. DDD No. of Dolphins versus Buffer size 10

Figure 5b. DDD No. of Dolphins versus Buffer size

西
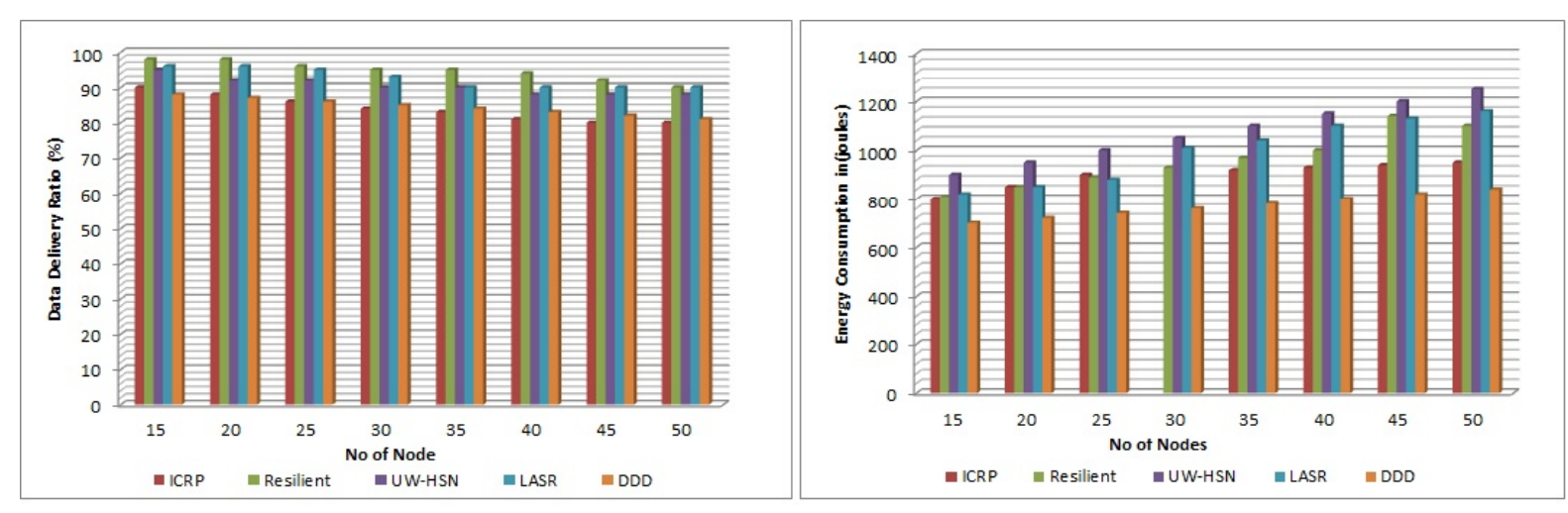

Figure 6. No. of Nodes versus Data Delivery Ratio

Figure 7. No. of Nodes versus Energy Consumption

\section{OPEN RESEARCH ISSUES}

Open research issues for the designing of reliable communication in underwater environment are:

a. In underwater environment the available bandwidth is limited; so the design of reliable communication routing protocol must be designed in such a way; which can show the better results on available acoustic channel bandwidth [19].

b. Acoustic channel severely impaired with multipath and fading problem, so design of routing protocol must consider such kind of acoustic channel problems [20].

c. Propagation delay is also a major issue in underwater environment because the acoustic channel's magnitude is five orders higher than radio signalling [11].

d. In underwater environment the design of routing protocol also face the high bit error rate and loss of connectivity due to extreme characteristics of acoustic channel [21].

e. Normal terrestrial network sensor batteries are not feasible for underwater environment and batteries cannot easily be recharged in underwater environment; so design of high power batteries and recharge of batteries in underwater environment is also a major issue [22]. 
f. Due to the fouling and corrosions in underwater environment the sensor nodes can be prone [23].

\section{CONCLUSION}

In this paper, we have described the overview of the data forwarding routing protocols based on single path. We have focused the designing issues of the routing protocols like deployment, localization, data forwarding, and route maintenance. We discussed the simulation scenarios of the single path routing protocols with their critical analysis and problems. We also presented the open research issues in underwater environment. The definitive objective of this research article is to inspire the researchers for more research efforts in the field of the underwater routing protocols.

\section{REFERENCES}

[1] J. Shen, et al., "A novel routing protocol providing good transmission reliability in underwater sensor networks," Journal of Internet Technology, vol. 16, pp. 171-178, 2015.

[2] B. Namazi and K. Faez, "Energy-Efficient multi-speed routing protocol for wireless sensor networks," International Journal of Electrical and Computer Engineering, vol. 3, pp. 246, 2013.

[3] A. P. Gopi, et al., "Designing an Adversarial Model Against Reactive and Proactive Routing Protocols in MANETS: A Comparative Performance Study," International Journal of Electrical and Computer Engineering, vol. 5, 2015.

[4] S. Umar, et al., "Tree Based Energy Balancing Routing Protocol by Self Organizing in Wireless Sensor Networks," International Journal of Electrical and Computer Engineering, vol. 5, 2015.

[5] S. M. Jani, et al., "Designing and Simulation of Surrounding Supporting Multicast Routing Protocol," International Journal of Electrical and Computer Engineering (IJECE), vol. 6, 2015.

[6] K. N. Qureshi, et al., "Geographical Forwarding Methods in Vehicular Ad hoc Networks," International Journal of Electrical and Computer Engineering, vol. 5, 2015.

[7] G. Han, et al., "Routing protocols for underwater wireless sensor networks," Communications Magazine, IEEE, vol. 53, pp. 72-78, 2015.

[8] J. S. Kiran, et al., "Review on Underwater Sensor Networks: Applications, Research Challenges and Time Synchronization," in International Journal of Engineering Research and Technology, 2015.

[9] P. R. Jadhao and M. M. Ghonge, "Energy Efficient Routing Protocols For Underwater Sensor Networks-A Survey," Energy, vol. 1, 2015.

[10] S. M. Ghoreyshi, et al., "A Novel Cooperative Opportunistic Routing Scheme for Underwater Sensor Networks," Sensors, vol. 16, pp. 297, 2016.

[11] A. Wahid, et al., "MRP: A Localization-Free Multi-Layered Routing Protocol for Underwater Wireless Sensor Networks," Wireless Personal Communications, vol. 77, pp. 2997-3012, 2014.

[12] M. Ahmed and M. Salleh, "Localization schemes in Underwater Sensor Network (UWSN): A Survey," TELKOMNIKA Indonesian Journal of Electrical Engineering, vol. 17, 2015.

[13] W. Liang, et al., "Information-carrying based routing protocol for underwater acoustic sensor network," in Mechatronics and Automation, 2007. ICMA 2007. International Conference on, pp. 729-734, 2007.

[14] D. Pompili, et al., "A resilient routing algorithm for long-term applications in underwater sensor networks," in Proc. of Mediterranean Ad Hoc Networking Workshop (Med-Hoc-Net), 2006.

[15] K. Ali and H. Hassanein, "Underwater wireless hybrid sensor networks," in Computers and Communications, 2008. ISCC 2008. IEEE Symposium on, pp. 1166-1171, 2008.

[16] E. A. Carlson, et al., "Location-aware routing protocol for underwater acoustic networks," in OCEANS 2006, pp. 16, 2006.

[17] M. Ayaz, et al., "A survey on routing techniques in underwater wireless sensor networks," Journal of Network and Computer Applications, vol. 34, pp. 1908-1927, 2011.

[18] E. Magistretti, et al., "A mobile delay-tolerant approach to long-term energy-efficient underwater sensor networking," Wireless Communications and Networking Conference, WCNC 2007. IEEE, pp. 2866-2871, 2007.

[19] I. F. Akyildiz, et al., "Underwater acoustic sensor networks: research challenges," Ad hoc networks, vol. 3, pp. 257$279,2005$.

[20] R. Headrick and L. Freitag, "Growth of underwater communication technology in the US Navy," Communications Magazine, IEEE, vol. 47, pp. 80-82, 2009.

[21] N. Javaid, et al., "iAMCTD: Improved Adaptive Mobility of Courier Nodes in Threshold-Optimized DBR Protocol for Underwater Wireless Sensor Networks," International Journal of Distributed Sensor Networks, 2014.

[22] T. Ali, et al., "End-to-End Delay and Energy Efficient Routing Protocol for Underwater Wireless Sensor Networks," Wireless Personal Communications, vol. 79, pp. 339-361, 2014.

[23] S. Climent, et al., "Underwater acoustic wireless sensor networks: advances and future trends in physical, MAC and routing layers," Sensors, vol. 14, pp. 795-833, 2014. 